\title{
Resistance testing in children changing human immunodeficiency virus type 1 protease inhibitor
}

\author{
JEAN SERVAIS, MD, MARC HAINAUT, MD, VÉRONIQUE SCHMITZ, MD, PHILIP MAES, MD, \\ KATRIEN FRANSEN, PHD, DOLORES VAIRA, BS, BÉNÉDICTE BRICHARD, MD, VIC ARENDT, MD, \\ FRANÇOIS SCHNEIDER, MD, ROBERT HEMMER, MD AND JEAN-CLAUDE SCHMIT, MD, PHD
}

From Laboratoire de Rétrovirologie, Centre de Recherche Public-Santé, Luxembourg (JS, VA, FS, RH, JCS); and Service de Pédiatrie, Centre Hospitalier Universitaire Saint-Pierre $(\mathrm{MH})$, and Service de Pédiatrie, Cliniques Universitaires Saint-Luc, Brussels (BB), ${ }^{3}$ Service de Pédiatrie, Hôpital de la Citadelle (VS), and AIDS Reference Laboratory, Centre Hospitalier Universitaire (DV), Liège (DV), and Koningin Paola Ziekenhuis, Academisch Ziekenhuis Middelheim (PM), and AIDS Reference Laboratory, Institute of Tropical Medicine (KF), Antwerp, Belgium.

\begin{abstract}
Objective. To assess genotypic and phenotypic resistance testing in HIV-1-infected children failing a first protease inhibitor (PI) therapy.

Methods. In a multicenter observational study 21 children, ages 3 to 16 years, were given two reverse transcriptase inhibitors and one PI (mainly ritonavir, $n=18$ ). They were subsequently treated with single or dual Pi-based therapy (predominantly nelfinavir, $n=10$, or ritonavir-saquinavir, $n=7$ ). Resistance testing was performed at the time of therapy switch via direct sequencing and a recombinant virus susceptibility assay.

Results. A total of 21 genotypic and 15 phenotypic resistance profiles were obtained. Most viruses displayed several reverse transcriptase mutations; however, 7 isolates maintained a wild-type protease. Ritonavir targeted the well-known pathway containing 82, 54, 46 and other secondary (nonactive site) mutations including $T 74 A$. No in vitro cross-resistance, i.e. $\geq 8$-fold resistance to saquinavir or amprenavir, was encountered. Secondary mutations enhanced the prediction of ritonavir resistance (i.e. L1OI) and in vitro nelfinavir cross-resistance (i.e. $K 20 R / I$ ) conferred by primary (active site) resistance mutations. Either the 82, 54, 46 mutational genotype or the phenotype showing $\geq 8$-fold nelfinavir cross-resistance predicted a poorer virologic response to nelfinavir salvage therapy.

Conclusion. In a small cohort of heavily pre-treated pediatric patients, resistance testing appears to predict the response to nelfinavir as salvage for a ritonavir-based therapy. This is further supported by the correlation between ritonavir-selected mutations and in vitro nelfinavir cross-resistance. Prospective studies should assess clinical outcome in children undergoing regimen changes based on resistance testing.
\end{abstract}

Key words: Human immunodeficiency virus type 1-infected children, protease inhibitor resistance, nelfinavir, highly active antiretroviral therapy, cross-resistance.

\section{INTRODUCTION}

Although a range of antiretroviral drugs is now available for use in children, the most appropriate pediatric treatment regimens remain to be defined. Recently an investigation on therapeutic practices in Europe showed that the availability of protease inhibitors (PI) varied between countries and that initiation of antiretroviral therapy in children was often delayed until evidence of clinical disease progression. Policies also varied for second line therapies with regard to progression, side effects and drug resistance. ${ }^{1}$ 
Pi-based highly active antiretroviral therapy (HAART) improves the virologic, immunologic and growth status ${ }^{2}$ and was found to be more effective than a combination of two nucleoside reverse transcriptase inhibitors (NRTI). ${ }^{3}$ In addition immune restoration with HAART was better in children than in adults, probably because of a greater thymic activity. ${ }^{2}$ In NRTI-treated children with detectable viral load, a change to a Pi-containing HAART, either with nelfinavir (NFV) or ritonavir (RTV) resulted in a response rate [viral load (VL) $<400$ copies/ml] of $\sim 25$ to $50 \%$. ${ }^{2,4}$ Salvage therapy including two PI demonstrated a limited long term effectiveness, with the combination RTV-saquinavir (SQV) doing slightly better than NFV-SQV. ${ }^{5}$ The extent to which this poor response is caused by viral resistance is unknown. In addition other factors may be associated in some instances with a poorer response such as a prior exposure to NRTI, a high initial viral load, low CD4 counts and a more advanced stage of disease. ${ }^{6}$ Under both in vitro and in vivo conditions, more or less specific mutation patterns have been associated with a reduced sensitivity to different PI. Significant levels of PI resistance appear only after several mutations have accumulated. ${ }^{7}$ Cross-resistance among PI is commonly encountered, ${ }^{8}$ thus substantially reducing subsequent treatment options. In adults resistance testing predicts second line treatment outcome in observational and intervention-based studies. ${ }^{9,10}$

In children drug tolerability, availability of appropriate formulations and dosages, and viral resistance reduce therapeutic choices. In the medical literature longitudinal data on HIV resistance and viral or immune markers in HIV-infected children are limited to date.

For these reasons we assessed the use of genotypic and phenotypic resistance testing in Pi-treated children. We evaluated the extent to which resistance-related mutations were present in children switching from a first to a second Pi-based HAART. We also tested whether viral genotypes correlated with phenotypic PI resistance. We furthermore evaluated in vitro PI cross-resistance and studied the association between resistance and second line treatment outcome.

\section{MATERIALS AND METHODS}

\section{Patients.}

Twenty-one HIV-1-infected children with virologic failure to a first Pi-based HAART were included in a Belgian multicenter observational study. Virologic failure was defined as a $<1 \log 10$ decrease in viral load compared with pretreatment values. Children were recruited at the Centre Hospitalier Univer-sitaire Saint-Pierre, Brussels $(n=12)$, the Hôpital de la Citadelle, Liège $(n=4)$, the Koningin Paola Zieken-huis, Antwerp $(n=4)$ and the Cliniques Universitaires Saint-Luc, Brussels $(n=1)$. Baseline data were obtained at the time of initiation of a second line PI-containing HAART. All children had acquired HIV through vertical transmission, and a majority had advanced disease. Their ages ranged from 3 to 16 years. A total of 18 were of African origin, and 12 were female. Eleven children were CDC clinical Stage C and CD4 Stage $3 .^{11}$ The median CD4 percentage was 15 (interquartile, 5 to 23 ), whereas the mean baseline viral load was $5.22 \pm 0.67 \log _{10} \operatorname{copies} / \mathrm{ml}$. Four were infected with clade $\mathrm{A}$ or $\mathrm{G}$ and one with clade $\mathrm{C}$ or $\mathrm{F}$ or $\mathrm{J}$ virus.

\section{Treatment.}

Twenty of 21 patients were pretreated with NRTI before HAART with a median number of 3 drugs (range, 2.25 to 3.00) and a median duration of exposure of 26.5 months (range, 12 to 30.5). First line HAART regimens consisted of 2 NRTI and a single PI [predominantly RTV (Table 1)] with a median duration of treatment of 17.9 months (quartiles, 7.6 to 23 months). Second line HAART, with a median duration of 21.8 months (11.7 to 24.8 months), was based on at least 2 NRTI and single or dual PI [mainly NFV and to a lesser extent RTV-SQV (Table 1)] and/or nevirapine (NVP, $n=6$ ). Anti-HIV treatment was changed according to the standards of care, but without access to resistance testing.

\section{Samples.}

Blood samples taken between June, 1997, and April, 2000, were batch-tested for drug resistance at the end of the study period. Samples were originally obtained for viral load and CD4 measurements during the 3-monthly clinical evaluations and whenever a major clinical event occurred. They were analyzed by direct sequencing of the protease $(\mathrm{PR})$ and reverse transcriptase $(\mathrm{RT})$ coding regions of pol $(n=21)$ and were submitted to phenotypic PI resistance testing for indinavir (IDV), RTV, SQV, NFV and amprenavir (APV). Operators performing and interpreting the genotypic assay were unaware of both clinical data and phenotypic susceptibility. 


\section{Nucleic acid extraction and DNA sequencing.}

Viral RNA was extracted from $140 \mu$ l of EDTA-plasma with the use of the QIAamp viral RNA kit (Qiagen, Hilden, Germany). Sequencing of the complete PR-coding regions of pol and nucleotides 111 to 741 of the RT was performed by the TruGene HIV-1 assay (Visible Genetics, Toronto, Canada). All sequences were compared with HГV-1 LAV-1 (GenBank/EMBL Accession No. K02013), submitted to the GenBank/EMBL databases and are available under Accession Nos. AJ297110 to AJ297130 and AJ297132 to AJ297152. Subtyping was performed by submission of the PR and RT sequences to the HIV subtyping tool (located at http://www.ncbi.nlm.nih.gov/retroviruses/subtype/ subtype.html) of the National Center for Biotechnology Information, Bethesda, MD. Drug resistance mutations were differentiated into primary and secondary according to the consensus statement of the International AIDS Society. ${ }^{12}$

\section{Phenotypic drug resistance.}

A replicating virus was obtained through homologous recombination of a PR-deleted, $\mathrm{HIV}-\mathrm{l}_{\mathrm{HXB} 2}$-derived provirus (Glaxo Wellcome Research and Development, Ware, UK) with the PCR amplification product (643 bp) containing the PR-coding regions of pol from a clinical isolate. Primers and assay conditions were previously described. ${ }^{13}$ The recombinant virus was titrated for infectivity and used in drug susceptibility assays. Briefly, seven 3 -fold dilutions of drugs were tested in triplicates. The median 50\% inhibitory concentration was calculated using the median-effect equation. ${ }^{14}$ Results were expressed as fold-resistance compared with the wildtype reference strain HIV $-1_{\text {IIIB }}$, tested in 2 triplicates within the same run. For the wild-type reference, the median 50\% inhibitory concentrations from 6 independent runs were: IDV, $166 \mathrm{nM}$ (range, 64 to 191); RTV, 23 $\mathrm{nM}$ (range, 4 to 47); SQV, $7 \mathrm{nM}$ (range, 3 to 8); NFV, $24 \mathrm{nM}$ (range, 12 to 35); and APV, $30 \mathrm{nM}$ (range, 14 to 35). IDV was provided by Merck (West Point, PA), RTV by Abbott (Abbott Park, IL), SQV by Roche (Welwyn, UK), NFV by Agouron (San Diego, CA) and APV by Glaxo-Wellcome (Erembodegem, Belgium).

\section{Plasma viral load.}

HIV-1 plasma viral load was measured by the Amplicor HIV-1 Monitor Kit 1.5 (Roche, Brussels, Belgium) with a detection limit of 50 copies $/ \mathrm{ml}$.

\section{Study variables related to resistance assays.}

Phenotypic resistance was either assessed as a dependent (continuous) variable for its association with resistance mutations and as a (ordinal) predictor variable of viral load changes in accordance with the study objectives. When a dependent variable, $\log _{10}$ transformation of the fold resistance values was used for the normality of the distribution, whereas a three level ordinal variable was used conventionally to assess the phenotype as a predictor of viral load changes to facilitate the clinical interpretation. It was set as resistant ( $\geq 8$-fold, scored as 1$)$, intermediate (4- to 8-fold, scored as 0.5 ) and sensitive ( $<4$-fold, scored as 0$)$. The quasispecies phenomenon allows a three level scoring system for mutations, given that the detection of mixtures by direct sequencing is rather qualitative: 1 for mutant; 0 for wild-type codons; and 0.5 for mixtures of both.

\section{Statistics.}

The association between mutations (either the nature or the number) and the magnitude of resistance or the changes in viral load was measured by the slope coefficient of a linear regression. Each PR mutation was assessed in simple and stepwise regression, because some so-called secondary mutations could have an impact on the resistance only in the presence of primary resistance mutations. ${ }^{15}$ The $P$ values resulted from an $F$ test for the multiple correlation coefficient squared $\left(R^{2}\right)$ change and were adjusted in a forward stepwise multiple regression with an alpha value for entry of 0.05 and 0.10 for deletion. $R^{2}$ indicated the percentage of variance in dependent variable magnitude explained by the predictors. The minimum number of observations for a predictor to fit the multiple regression models was set to 5. The Pearson correlation coefficient, $r$, was used for the correlation between levels of resistance. All calculations were made with the SPSS 9.0 statistical software (SPSS, Chicago, IL, 1999). 


\section{RESULTS}

Genotype was obtained for all 21 patients. For 2 patients with a first negative genotype (Patients 10 and 20), additional samples were used (Table 1). Fifteen strains, including 2 subtype G viruses, yielded a susceptibility phenotype.

\section{Failure of first line Pi-based therapy.}

At the moment children were changed to a second PI, 7 of 21 virus isolates did not have primary PI resistance mutations and 4 of 15 remained phenotypically susceptible to all PI. The protease mutations for nelfinavir (D30N), saquinavir (G48V) and amprenavir (I50V) were not encountered (Table 1). From 2 IDV-treated patients only Patient 3 harbored virus with IDV resistance mutations, resulting in a 7-fold cross-resistance to RTV. From the $18 \mathrm{RTV}$-treated patients, 5 harbored virus with wild-type protease but included polymorphisms at codons L10I or V $(n=4$ of 5$), \mathrm{K} 20 \mathrm{M}(n=1$ of 5$), \mathrm{L} 63 \mathrm{P}$ or V $(n=2$ of 5$)$ or at the location E35D-M36I-S37N $(n=4$ of 5). The remaining 13 patients presented primary resistance mutations at codons 82,54 and 90 (Fig. 1). Interestingly the unusual T74A or S substitutions were observed in 7 children failing RTV treatment without relation to a particular subtype.

In 10 children failing RTV and harboring viruses with an I54V, mutations at codon 82 were always found. M46I or L was mainly detected as a wild-type/ mutant mixture (3 of 4 cases) and associated with high level resistance. Primary RT mutations, predominantly T215Y or F and M184V, were detected in 20 patients at the frequency of 1 to 8 mutations per isolate. The non-NRTI-related mutations Y181C and K103N were also encountered (Table $1)$.

\section{Drug resistance pathways and phenotypic drug susceptibility.}

No strains were resistant (i.e. $\geq 8$-fold resistant) to SQV or APV (Table 1). Resistance to other PI was only encountered in children failing RTV treatment. Interpretable phenotypes were obtained in 9 of the 13 children failing RTV treatment with resistance mutations in their viruses (Table 1).

In simple regression the PR mutations significantly associated with increased resistance to RTV were located at codons 82, 54 and 74 (Table 2). In stepwise multiple regression the variance in RTV resistance rose from 46 to $65 \%$ when L10I was included in the 82/54 mutations pattern. In simple regression there was a relationship between the presence of the I54V mutation and a loss of susceptibility to NFV (Table 2). I54V, linked to V82A, was entered first in stepwise regression and explained $34 \%$ of the variance in NFV cross-resistance magnitude (Table 2). K20R or I, entered second, added a further $23 \%$ (Table 2).

All levels of resistance were correlated in pairwise comparisons $(r \geq 0.58$, df $=13, P<0.005)$. RTV and NFV levels of resistance were particularly well correlated $(r=0.80, \mathrm{df}=13, P<0.001)$. 
TABLE 1. Treatment and drug resistance in children

\begin{tabular}{|c|c|c|c|c|c|c|c|c|c|c|c|c|c|}
\hline Patient & $\begin{array}{c}\text { First } \\
\text { PI }\end{array}$ & Protease Mutations & $\begin{array}{c}\text { IDV } \\
\text { (Fold) }\end{array}$ & $\begin{array}{l}\text { RTV } \\
\text { (Fold) }\end{array}$ & $\begin{array}{c}\text { SQV } \\
\text { (Fold) }\end{array}$ & $\begin{array}{l}\text { NFV } \\
\text { (Fold) }\end{array}$ & $\begin{array}{c}\text { APV } \\
\text { (Fold) }\end{array}$ & First RTI & RT Mutations & Second PI & Second RTI & $\Delta \mathbf{V L}$ & Adherence* $^{*}$ \\
\hline 1 & $\mathrm{NFV}$ & $37 N, 63 P, 77 I, 88 S \dagger$ & 3 & 1 & 1 & 3 & 1 & $\mathrm{ABC}+\mathrm{NVP}$ & $\begin{array}{l}41 \mathrm{~L}, 44 \mathrm{D}, 67 \mathrm{~N}, 70 \mathrm{R}, 103 \mathrm{~N}, 118 \mathrm{I}, \\
181 \mathrm{C}, 184 \mathrm{~V}, 215 \mathrm{Y}\end{array}$ & RTV/SQV & $\mathrm{d} 4 \mathrm{~T}+\mathrm{ddI}+\mathrm{HU}$ & -1.54 & No \\
\hline 2 & IDV & Wild typet & 1 & 1 & 1 & 1 & 1 & $\mathrm{ZDV}+3 \mathrm{TC}$ & $41 L, 67 N, 69 D, 70 R, 215 Y$ & RTV & $\mathrm{d} 4 \mathrm{~T}+3 \mathrm{TC}$ & -3.61 & Yes \\
\hline 3 & IDV & $20 R, 36 I, 37 N, 46 I, 82 A$ & 3 & 7 & 1 & 1 & 1 & $\mathrm{ZDV}+\mathrm{ddI}$ & $67 N, 70 R, 118 I, 215 Y$ & RTV/SQV & $\mathrm{d} 4 \mathrm{~T}+3 \mathrm{TC}$ & -0.07 & No \\
\hline 4 & RTV & $10 \mathrm{~V}, 34 \mathrm{D}, 35 \mathrm{D}, 36 \mathrm{I}^{\dagger}$ & & & & & & $\mathrm{ZDV}+3 \mathrm{TC}$ & $69 \mathrm{D}, 70 \mathrm{R}, 184 \mathrm{~V}$ & RTV/SQV & $\mathrm{d} 4 \mathrm{~T}+\mathrm{ddI}+\mathrm{HU}$ & -1.18 & No \\
\hline 5 & RTV & $10 \mathrm{~V}, 20 \mathrm{M}, 36 \mathrm{I}-37 \mathrm{N \dagger}$ & & & & & & $\mathrm{ZDV}+3 \mathrm{TC}$ & Wild type & NFV & $\mathrm{ZDV}+\mathrm{NVP}$ & -2.59 & Yes \\
\hline 6 & RTV & $10 \mathrm{I}, 36 \mathrm{I}, 37 \mathrm{~N}, 63 \mathrm{V \dagger}$ & 1 & 1 & 1 & 1 & 1 & $\mathrm{ZDV}+\mathrm{ddI}+\mathrm{NVP}$ & $41 L, 67 N, 69 D, 181 C, 215 Y$ & NFV & $\mathrm{ZDV}+3 \mathrm{TC}$ & -2.80 & No \\
\hline 7 & RTV & $63 P \dagger$ & 1 & 1 & 1 & 1 & 1 & $\mathrm{ZDV}+3 \mathrm{TC}$ & $\begin{array}{l}41 \mathrm{~L}, 44 \mathrm{D}, 67 \mathrm{~N}, 69 \mathrm{D}, 74 \mathrm{~V}, 103 \mathrm{~N} \\
118 \mathrm{I}, 181 \mathrm{C}, 215 \mathrm{Y}\end{array}$ & NFV & $\mathrm{d} 4 \mathrm{~T}+\mathrm{ddI}$ & -2.50 & Yes \\
\hline 8 & RTV & $10 I, 35 D, 36 I, 37 N \dagger$ & 1 & 7 & 1 & 1 & 1 & $\mathrm{ZDV}+3 \mathrm{TC}$ & $41 L$ & RTV & $\mathrm{ZDV}+3 \mathrm{TC}$ & -2.18 & Yes \\
\hline 9 & RTV & $32 V / I, 46 M / I, 54 I / V, 63 P, 82 A, 93 L$ & & & & & & $\mathrm{~d} 4 \mathrm{~T}+\mathrm{ddI}$ & $41 L, 215 Y$ & RTV/SQV & $\mathrm{d} 4 \mathrm{~T}+\mathrm{ddI}+\mathrm{HU}$ & -0.03 & No \\
\hline 10 & RTV & $\begin{array}{l}10 I, 33 F, 35 D, 36 I, 37 N, 54 V, 63 T, \\
82 \mathrm{~A}\end{array}$ & & & & & & $\mathrm{~d} 4 \mathrm{~T}+3 \mathrm{TC}$ & $184 \mathrm{~V}$ & NFV & $\mathrm{d} 4 \mathrm{~T}+\mathrm{NVP}+\mathrm{ABC}$ & -1.13 & Yes \\
\hline 11 & RTV & $10 I, 35 D-37 N, 54 V, 63 P, 82 A, 90 M$ & & & & & & $\mathrm{ZDV}+3 \mathrm{TC}$ & $41 L, 69 D, 70 R, 184 V, 215 Y$ & NFV & $\mathrm{ZDV}+3 \mathrm{TC}$ & -1.50 & Yes \\
\hline 12 & RTV & $33 F, 36 I, 37 N, 54 V, 63 T, 74 A, 82 A, 93 T$ & & & & & & $\mathrm{ZDV}+3 \mathrm{TC}$ & $41 L, 184 \mathrm{~V}, 215 \mathrm{Y}$ & NFV & $\mathrm{d} 4 \mathrm{~T}+\mathrm{ddI}$ & -0.89 & Yes \\
\hline 13 & RTV & $10 I, 33 F, 35 D, 37 N, 54 V, 63 R, 82 A$ & 2 & 4 & 1 & 2 & 1 & $\mathrm{ZDV}+3 \mathrm{TC}$ & Wild type & RTV/SQV & $\mathrm{d} 4 \mathrm{~T}+\mathrm{ddI}+\mathrm{HU}$ & -0.58 & No \\
\hline 14 & RTV & $20 I, 36 I, 37 N, 82 I, 90 M$ & 1 & 5 & 1 & 5 & 1 & $3 \mathrm{TC}+\mathrm{ddI}$ & $41 L, 67 N, 69 D, 184 V, 215 Y$ & NFV & $\mathrm{ZDV}+\mathrm{NVP}+\mathrm{PFA}$ & -2.96 & Yes \\
\hline 15 & RTV & $35 D, 36 I, 37 N, 46 M / L, 74 A, 82 F, 90 M$ & 1 & 27 & 2 & 2 & 1 & $\mathrm{~d} 4 \mathrm{~T}+3 \mathrm{TC}$ & $41 L, 44 D, 67 N, 118 I, 184 V, 215 Y$ & NFV/SQV & $\mathrm{ddI}+\mathrm{HU}+\mathrm{ABC}$ & -2.38 & Yes \\
\hline 16 & RTV & $20 I, 33 F, 35 D, 36 I, 37 N, 74 T / A, 82 S$ & 1 & 50 & 1 & 2 & 1 & $\mathrm{~d} 4 \mathrm{~T}+3 \mathrm{TC}$ & $215 Y$ & RTV/SQV & $\mathrm{NVP}+\mathrm{ABC}$ & -4.48 & No \\
\hline 17 & RTV & $10 I, 20 R, 82 A, 54 V, 63 P$ & 4 & 62 & 1 & 7 & 2 & $\mathrm{~d} 4 \mathrm{~T}+3 \mathrm{TC}$ & $184 \mathrm{~V}, 215 \mathrm{Y}$ & NFV/SQV & $\mathrm{ddI}+\mathrm{HU}+\mathrm{ABC}$ & -1.30 & Yes \\
\hline 18 & RTV & $35 \mathrm{D}, 36 \mathrm{I}, 37 \mathrm{~N}, 54 \mathrm{~V}, 74 \mathrm{~S}, 82 \mathrm{M}, 90 \mathrm{M}, 93 \mathrm{~L}$ & 8 & 63 & 3 & 9 & 3 & $\mathrm{ZDV}+3 \mathrm{TC}$ & $41 L, 69 D, 70 R, 215 Y$ & RTV/SQV & $\mathrm{d} 4 \mathrm{~T}+\mathrm{ddI}+\mathrm{HU}$ & 0.37 & No \\
\hline 19 & RTV & $\begin{array}{l}10 I, 20 I, 36 I, 37 N, 54 I / V, 63 L / P, 74 T / S, \\
82 I / S\end{array}$ & 5 & 188 & 3 & 21 & 2 & $\mathrm{~d} 4 \mathrm{~T}+3 \mathrm{TC}$ & $41 L, 44 D, 69 D, 74 V, 118 I$ & NFV & $\mathrm{d} 4 \mathrm{~T}+\mathrm{ddI}+\mathrm{NVP}$ & -0.62 & No \\
\hline 20 & RTV & $10 I, 33 F, 54 V, 74 A, 82 A$ & 1 & 393 & 1 & 13 & 1 & $\mathrm{ZDV}+3 \mathrm{TC}+\mathrm{NVP}$ & $70 \mathrm{R}, 181 \mathrm{C}, 184 \mathrm{~V}$ & NFV & $\mathrm{d} 4 \mathrm{~T}+\mathrm{ddI}+\mathrm{NVP}$ & 0.15 & No \\
\hline 21 & RTV & $10 I, 20 I, 46 M / I, 54 I / V, 82 \mathrm{~V} / F$ & 40 & 395 & 2 & 22 & 6 & $\mathrm{~d} 4 \mathrm{~T}+\mathrm{ddI}$ & $41 L, 67 N, 69 D, 70 R, 215 Y$ & NFV & $\mathrm{d} 4 \mathrm{~T}+\mathrm{ABC}$ & -0.82 & Yes \\
\hline
\end{tabular}

* Adherence was also assessed by the physician set as constant (yes) or no.

$\dagger$ Wild-type or polymorphisms only.

ZDV, zidovudine; d4T, stavudine; ddl, didanosine; 3TC, amivudine; ABC, abacavir; NVP, nevirapine; HU, hydroxy-urea; PFA, foscarnet; $\Delta \mathrm{VL}$, changes in viral load compared to baseline: $\Delta \mathrm{log}_{10}$ copies/ml. 


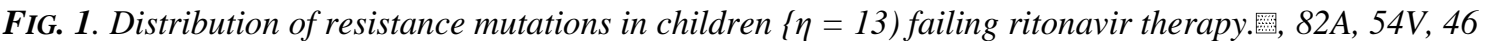
$M / 1$;圂, $82 F, 54 I / N, 46 \mathrm{M} / \mathrm{L} ; \mathbf{\square}, 82 \mathrm{~S}$;目, 82I/M.

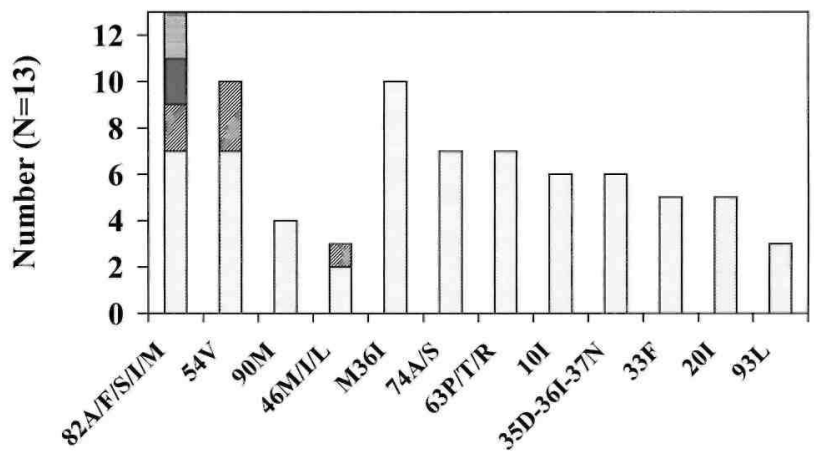

Mutations

Second line Pi-based therapy. Seven patients failed $(<1 \log 10$ VL decrease) salvage therapy (Table 1). Patient 1 with first line NFV, presenting wild-type PR and RT-resistant virus, responded to RTV-SQV-based salvage therapy (cf. Table 1). Patient 2, after first line IDV therapy, achieved a >2 log response to RTV therapy with wild-type PR and numerous RT mutations (Table 1).

From the 18 children with first line RTV therapy, 10 were subsequently changed to NFV-based therapy. Three patients with wild-type PR viral strains (Patients 5, 6 and 7) experienced a decrease in VL of at least 2 logs even though 2 harbored RT mutant viruses (Table 1). Patient 14, with PR 82/90 substitutions and multiple RT mutations and a 5-fold resistance to RTV and NFV, had a $>2$ log response and regained normal weight from less than percentile 3. Patients with $\geq 8$-fold NFV resistance induced by PR 82, 54, 10 and other secondary RTVselected mutations (Patients 19 to 21) failed on NFV-based therapy. For Patient 8 initially not responding to RTV therapy with mild toxicity, RTV was reintroduced and resulted in a $>1$ log response despite a 7-fold resistance linked to nonactive site mutations or polymorphisms.

Five patients were treated with RTV followed by RTV-SQV-based therapy. Three failed with inconstant adherence. They harbored RTV selected resistance mutations without in vitro cross-resistance to SQV. Patient 4 with a wild-type PR and with RT mutations had a response. Patient 16 with 50-fold resistance to RTV but susceptible to SQV obtained a $>2 \log$ response (Table 1).

Two patients were given NFV/SQV as salvage to RTV-based therapy. Patient 15 with a 27-fold resistance to RTV and a 2-fold resistance to SQV, and NFV had a >2 $\log$ response. Patient 17, with a 62-fold resistance to RTV and a 7-fold resistance to NFV but fully susceptible to SQV, had a -1.30 log response.

The virologic response was the median $\Delta \log _{10}$ viral load from baseline measured at Months 3, 6 and 9 . Viral load values reported as <50 copies $/ \mathrm{ml}$ were set to $25 \mathrm{copies} / \mathrm{ml}$ (for three patients), as previously suggested. ${ }^{16} \mathrm{In}$ simple linear regression a poorer response was only linked to the $I 54 \mathrm{~V}$ mutation (Table 3 ). This mutation explained $42 \%$ of the variance in virologic response. Linear regression could not identify any RT mutations significantly associated with virologic response. Other baseline characteristics that may impair the treatment response in some such as a higher clinical stage (scored 1 to 3), ${ }^{11}$ a lower CD4 percentage ${ }^{11}$ or a high baseline viral load $\left(\log _{10}\right.$ copies $\left./ \mathrm{ml}\right)$ was not associated with a poorer response. Various models summing the number of PI resistance mutations were assessed. There was a relationship between the number of mutations including positions 46, 48, 54, 82 and 90 but also 10 and a poorer response (Table 3). The extent of cross-resistance was defined as the number of PI (scored from 0 to 5) with a resistant (scored as 1) or an intermediate (scored as 0.5 ) phenotype. A significant relationship was also noted between the extent of cross-resistance and a poorer response. 
In a subset analysis of NFV-treated patients, $I 54 \mathrm{~V}$ was included first and explained $81 \%$ of the variance in response to NFV-based HAART, T74A second explained a further $10 \%$ of the variance but the prevalence of this mutation was too small to allow inference $(n=3)$. There was a relationship between the number of mutations including positions 46, 54 and 82 but also 74 and a poorer response to NFV-containing therapy (Table $3)$. In simple regression NFV resistance, as an ordinal variable, was also a predictor of poorer response to NFV (Table 3).

\section{DISCUSSION}

In a cohort of mainly RTV-treated and NRTI-experienced children, one-third of the failing patients still harbored virus with wild-type protease. One can speculate that incomplete drug absorption in the presence of good adherence for several months would probably have induced viral resistance. Thus the absence of resistance mutations in the protease most likely means a poor adherence to the PI-containing regimen. Other explanations involve inadequate drug concentrations (pharmacokinetic reasons, limited drug potency) or cellular resistance (e.g. phosphoglycoprotein). Therefore resistance testing may allow differentiation between drug resistancerelated failure and failures of other origins. In addition some children with inconstant adherence to first line therapy responded to second line treatment. As shown previously ${ }^{17}$ the convenience for the patient of a particular drug regimen can play a major role in adherence.

Treatment failure was mainly linked to viral resistance in our study. Most of the children were heavily exposed to NRTI before the first PI was administered, and their viruses displayed multiple primary RT mutations. RTV selected mutations were similar to those observed in adults as previously shown ${ }^{18}$ but did not include $I 84 \mathrm{~V}$ or $A 71 V$ or $T$ mutations at the time of first drug failure. Until now the $T 74 A$ substitution has not been reported in adult patients failing RTV, but it was correlated to RTV resistance. $M 46 I$ or $L$ was mostly present as a mixture with wild-type virus suggesting a poor in vivo tolerance of this substitution as already observed in adults. ${ }^{19}$ Some secondary mutations known as polymorphisms, ${ }^{20,21}$ as for instance L10I, enhanced the prediction of RTV resistance magnitude based on the classical resistance pattern $\mathrm{V} 82 \mathrm{~A}$ and $I 54 \mathrm{~V}{ }^{7}$ In addition $\mathrm{K} 20 \mathrm{R}$ or I amplified the NFV cross-resistance conferred by the RTV-induced I54V. Cross-resistance to IDV was mediated by the RTV-selected M46I. These data corroborate observation on drug resistance in adult patients.

In our study either a genotype associating mutations at codons 82,54 and 46 or phenotypic cross-resistance to NFV predicted a poor response to NFV-based salvage therapy. This observation is suggestive of a RTV-induced NFV resistance pattern, lacking the typical NFV-selected D30N. Previous reports ${ }^{9}$ showed that PI resistance testing predicts second line treatment outcome in adults, suggesting that the PI component accounts for most of the benefit of combination therapy in NRTI-experienced patients. In our small pediatric study, the only predictors of response were the number of protease mutations (including L1OI) and the degree of crossresistance, mainly to NFV. I54V had a major contribution. In contrast the disease stage and the initial viral load were not predictive of response. However, in a larger study with first line NFV therapy, a sustained response was best achieved in children with high baseline lymphocyte counts, and failure was linked to $D 30 N^{22}$

As a consequence of the limited sample size, the statistical inferences must be confirmed in larger studies. Present PCR-based assays poorly discriminate for minor mutants, thus limiting the negative predictive value of resistance testing. In addition archived viruses, for instance in proviral lymphocyte DNA, may influence treatment outcome when recycling drugs. Moreover our study has the limitations of observational studies lacking both drug level monitoring.

In conclusion resistance testing in heavily pretreated pediatric patients predicts a poorer response to NFV as salvage to RTV based therapy even in a small sample size. The correlation between RTV-selected mutations and NFV in vitro cross-resistance further supports this assumption. Larger, prospective studies should assess children undergoing regimen changes based on resistance profiles. 
TABLE 2. Association between mutations and resistance magnitude. The association between protease mutations scored as 1 for mutant, 0.5 for wild-type/mutant mixture and 0 for wild-type, and $\log _{10}$ fold-resistance increase was measured by the slope of a linear regression. The $P$ values resulted from a $F$ test for $R^{2}$ change and were adjusted in stepwise multiple regression including all mutations that showed the best predictors explaining the variance $\left(R^{2}\right)$ in resistance magnitude in an ordered manner. The stronger predictor was entered first. The second explained a further contribution in variance. The reported numbers $(N)$ are first the wild-type, then the wild-type/mutant, then the mutant strains.

\begin{tabular}{|c|c|c|}
\hline \multirow{2}{*}{ Analysis Mutations } & \multicolumn{2}{|c|}{ Slope, $R^{2}$ change, $F$ change, $P$} \\
\hline & Ritonavir & Nelfinavir \\
\hline \multicolumn{3}{|l|}{ Simple regression $(\mathrm{df} 1=1, \mathrm{df} 2=13)$} \\
\hline$I 54 V(N=9 / 2 / 4)$ & $1.451,0.0416,8.539,0.013$ & $0.687,0.335,6.044,0.030$ \\
\hline$T 74 A / S(N=10 / 2 / 3)$ & $1.385,0.379,7.312,0.019$ & \\
\hline$V 82 A / F / S / I / M(N=5 / 1 / 9)$ & $1.328,0.456,10.069,0.008$ & \\
\hline \multicolumn{3}{|c|}{ Stepwise regression $(\mathrm{df} 1=1, \mathrm{df} 2=13$ first, 12 second step) } \\
\hline$L 10 I / V / F(N=9 / 0 / 6)$ & $\begin{array}{l}0.825,0.195,6.149,0.031) \\
\text { Second }\left(R^{2}=0.651\right)\end{array}$ & \\
\hline$K 20 R / I(N=9 / 0 / 6)$ & & $\begin{array}{l}0.498,0.235,5.994,0.032) \\
\text { Second }\left(R^{2}=0.570\right)\end{array}$ \\
\hline$I 54 V(N=9 / 2 / 4)$ & & $\begin{array}{l}0.778,0.335,6.044,0.030 \\
\text { First }\left(R^{2}=0.335\right)\end{array}$ \\
\hline$V 82 A / F / S / I / M(N=5 / 1 / 9)$ & $\begin{array}{l}1.366,0.456,10.069,0.008 \\
\text { First }\left(R^{2}=0.456\right)\end{array}$ & \\
\hline
\end{tabular}

df, degree of freedom.

TABLE 3. Association between mutations and virologic response (simple linear regression) The association between virologic response (i.e. the median $\Delta \log _{10}$ viral load from baseline measured at Months 3, 6 and 9) and predictors (resistance data, baseline characteristics) was measured by the slope of a simple linear regression. Mutations were scored as 1 for mutant, 0.5 for wild-type/mutant mixture and 0 for wild-type. NFV resistance was scored as: $0,<4$-fold; $0.5,4$ - to 8 -fold; and 1 , $\geq 8$-fold. The extent of cross-resistance was defined as the number of PI (maximum 5) with resistance scored as 1 , or an intermediate phenotype scored as 0.5 . The $P$ values resulted from an $F$ test for $R^{2}$ change. Subset analyses were performed only on those patients treated with nelfinavir. Mutations: the reported numbers ( $N$ ) are first the wild-type, then the wild-type/mutant, then the mutant strains.

\begin{tabular}{|c|c|c|c|}
\hline \multirow{2}{*}{ Predictors } & \multicolumn{3}{|c|}{$\Delta \log _{10}$ HIV RNA Copies/ml } \\
\hline & Slope, $\Delta R^{2}$ & $\Delta F, P$ & $R^{2}$ \\
\hline \multicolumn{4}{|l|}{ All patients } \\
\hline$I 54 V(N=11 / 3 / 7)(\mathrm{df} 1=1, \mathrm{df} 2=19)$ & $1.890,0.418$ & $12.922,0.002$ & 0.418 \\
\hline Sum of protease mutations $(10,46,48,54,82,90)(N=21)(\mathrm{df} 1=1, \mathrm{df} 2=19)$ & $0.674,0.340$ & $9.263,0.007$ & 0.340 \\
\hline Extent of cross-resistance $(N=15)(\mathrm{df} 1=1, \mathrm{df} 2=13)$ & $0.730,0.324$ & $5.748,0.034$ & 0.324 \\
\hline \multicolumn{4}{|l|}{ Nelfinavir-treated patients } \\
\hline $\begin{array}{l}\text { Nelfinavir fold resistance (<4-fold, } N=2 ; 4 \text { - to } 8 \text {-fold, } N=1 \text {, }>8 \text {-fold, } N=3)(\mathrm{df} 1= \\
1, \text { df } 2=4)\end{array}$ & $2.319,0.743$ & $11.583,0.027$ & 0.743 \\
\hline$I 54 V(N=4 / 2 / 4)(\mathrm{df} 1=1, \mathrm{df} 2=8)$ & $1.954,0.662$ & $13.680,0.008$ & 0.662 \\
\hline Sum of protease mutations $(46,54,74,82)(N=10)(\mathrm{df} 1=1, \mathrm{df} 2=8)$ & $0.801,0.751$ & $24.181,0.001$ & 0.751 \\
\hline
\end{tabular}

df, degree of freedom. 


\section{ACKNOWLEDGMENTS}

This study was supported by the Fondation Recherche sur le SIDA, Luxembourg, the Centre de Recherche Public-Santé, Luxembourg, and a Bourse-Formation Recherche, Ministère de la Culture, de l'Enseignement Supérieur et de la Recherche, Luxembourg (BFR97/015).

\section{REFERENCES}

1. Bernardi S, Thorne C, Newell M, Giaquinto C, Tovo P, Rossi P. Variable use of therapeutic interventions for children wit human immunodeficiency virus type 1 infection in Europe. Eur J Pediatr 2000;159:170-5.

2. Essajee SM, Kim M, Gonzales C, et al. Immunologic and virologic responses to HAART in severely immunocompromised HIV-1 infected children. AIDS 1999;13:2523-32.

3. Nachman SA, Stanley K, Yogev R, et al. Nucleoside analogs plus ritonavir in stable antiretroviral therapy-experienced HIV-infected children: a randomized controlled trial. Pediatric AIDS Clinical Trials Group 338 Study Team. JAMA 2000;283:492-8.

4. Wiznia A, Stanley K, Krogstad P, et al. Combination nucleoside analog reverse transcriptase inhibitor(s) plus nevirapine, nelfinavir, or ritonavir in stable antiretroviral therapy-experienced HIV-infected children: week 24 results of a randomized controlled trial-PACTG 377. Pediatric AIDS Clinical Trials Group 377 Study Team. AIDS Res Hum Retroviruses 2000;16:1113-21.

5. Hoffmann F, Notheis G, Wintergerst U, Eberle J, Gürtler L, Belohradsky BH. Comparison of ritonavir plus saquinavir-and nelfinavir plus saquinavir-containing regimens as salvage therapy in children with human immunodeficiency type 1 infection. Pediatr Infect Dis $\mathbf{J}$ 2000;19:47-51

6. Ledergerber B, Egger M, Opravil M, et al. Clinical progression and virological failure on highly active antiretroviral therapy in HIV-1 patients: a prospective cohort study: Swiss HIV Cohort Study. Lancet 1999;353:863-8.

7. Molla A, Korneyeva M, Gao Q, et al. Ordered accumulation of mutations in HIV protease confers resistance to ritonavir. Nat Med $1996 ; 2: 760-6$

8. Condra JH, Schleif WA, Blahy OM, et al. In vivo emergence of HIV-1 variants resistant to multiple protease inhibitors. Nature 1995;374:569-71.

9. Zolopa AR, Shafer RW, Warford A, et al. HIV genotypic resistance patterns predict response to saquinavir-ritonavir therapy in patients in whom previous protease inhibitor therapy had failed. Ann Intern Med 1999;131:813-21.

10. Durant J, Clevenbergh P, Halfon P, et al. Drug-resistance genotyping in HIV-1 therapy: the VIRADAPT randomised controlled trial. Lancet 1999;353:2195-9.

11. Centers for Disease Control and Prevention. Revised classification system for human immunodeficiency virus infection in children $<13$ years of age. MMWR 1994;43:1-10.

12. Hirsch MS, Brun-Vézinet F, D'Aquila RT, et al. Antiretroviral drug resistance testing in adult HIV-1 infection, recommendations of an International AIDS Society-USA panel. JAMA 2000;283:2417-26.

13. Vandamme AM, Witvrouw M, Pannecouque C, et al. Evaluating clinical isolates for their phenotypic and genotypic resistance against anti-HIV drugs. In: Kinchington D, Schi-nazi RF, eds. Methods in molecular medicine, vol. 24. Totowa, NJ: Humana Press, 1999;19:223-58.

14. Chou TC. The median-effect principle and the combination index for quantitation of synergism and antagonism. In: Chou TC, Rideout DC, eds. Synergism and antagonism in chemotherapy. New York: Academic Press, 1991:61-102.

15. Condra JH, Holder DJ, Schleif WA, et al. Genetic correlates of in vivo viral resistance to indinavir, a human immunodeficiency virus type 1 protease inhibitor. J Virol 1996;70: 8270-6.

16. Gulick RM, Mellors JW, Havlir D, et al. Simultaneous vs. sequential initiation of therapy with indinavir, zidovudine, and lamivudine for HIV-1 infection: 100-week follow-up. JAMA 1998;280:35-41.

17. Becker S, Rachlis A, Gill J, et al. Successful substitution of protease inhibitors with efavirenz (EFV) in patients with undetectable viral loads: a prospective, randomized, multi-center, open-label study (DMP 049) [Abstract 20]. In: Eighth Conference on Retroviruses and Opportunistic Infections, Chicago, IL, February 4 to 8, 2001. Alexandria, VA: Foundation for Retrovirology and Human Health, 2001

18. Tamalet C, Yahi N, Thuret I, Michel G, Perrimond C. Mutation pattern of the RT and protease genes in HIV-infected children receiving combination therapy [Abstract 12257]. In: XIIth International AIDS Conference, Geneva, Switzerland, June 28 to July 3 . Produced by Multimedia in conjunction with Cicero, 1998:vol 12. 
Published in : Pediatric Infectious Disease Journal (2002)

Status : Postprint (Author's version)

19. Servais J, Lambert C, Fontaine E, et al. Comparison of DNA sequencing and a line probe assay for detection of human immunodeficiency virus type 1 in patients failing highly active antiretroviral therapy. J Clin Microbiol 2001;39: 454-9.

20. Kozal MJ, Shah N, Shen N, et al. Extensive polymorphisms observed in HIV-1 clade B protease gene using high-density oligonucleotide arrays. Nat Med 1996;2:753-9.

21. Servais J, Lambert C, Fontaine E, et al. Variant human immunodeficiency virus type 1 proteases and response to combination therapy including a protease inhibitor. Antimi-crob Agents Chemother 2001;45:893-900.

22. Krogstad P, Wiznia A, Luzuriaga K, et al. Treatment of human immunodeficiency virus 1-infected infants and children with the protease inhibitor nelfinavir mesylate. Clin Infect Dis 1999;28:1109-18. 